\title{
Reconstructing grains in 3D through 4D Scanning Precession Electron Diffraction
}

Patrick Harrison ${ }^{1}$, Xuyang Zhou ${ }^{2}$, Saurabh Mohan Das $^{3}$, Nicola Viganò ${ }^{4}$, Pierre Lhuissier ${ }^{1}$, Michael Herbig ${ }^{2}$, Wolfgang Ludwig ${ }^{5}$ and Edgar Rauch ${ }^{6}$

${ }^{1}$ SIMaP, CNRS/Grenoble INP, France, ${ }^{2}$ Max-Planck-Institut für Eisenforschung GmbH, Germany, ${ }^{3}$ MaxPlanck-Institut für Eisenforschung GmbH, Düsseldorf, Nordrhein-Westfalen, Germany, ${ }^{4}$ ESRF, France, ${ }^{5}$ MATEIS, INSA Lyon, United States, ${ }^{6}$ SIMaP, CNRS/Grenoble INP, United States

Scanning Precession Electron Diffraction (SPED) within the Transmission Electron Microscope (TEM) is a commonly employed technique used to identify crystalline phases and nanocrystallite orientation relationships within materials. In this technique a small electron probe is scanned over the sample and the electron diffraction pattern resulting from the local interaction between the probe and sample is recorded for each probe position, resulting in 4-dimensional data which contains both spatial and orientation information. Automated diffraction indexing algorithms through template matching ${ }^{1}$ provide excellent orientation mapping results, although, in the case of multiple overlapping nanocrystallites (grains), the indexing result is typically limited to the dominant grain. Recently developed multiple-indexing techniques may be used to index auxiliary grains ${ }^{2}$, however their complete spatial positions within the sample thickness cannot be distinguished from a single projection. The ability to determine orientation relationships between grains within the overall 3D material volume is necessary to fully understand the properties of materials ${ }^{3,4}$.

To this end we have performed few-tilt tomographic SPED to reconstruct phase and orientation information from an FeC sample, consisting of columnar ferrite grains with $30 \mathrm{~nm}$ diameter and cementite precipitates. SPED experiments were performed using a JEOL JEM-2200FS electron microscope operated at $200 \mathrm{kV}$ with $0.5^{\circ}$ precession angle. A Fischione Instruments Model 2050 On-Axis Rotation Tomography Holder was used to provide sample tilting and projections were acquired with a $30^{\circ}$ tilt step size. The quality of tomographic reconstructions typically scales with the number of projections, however experimental time is often a limiting factor for the microscope operator, so it is of interest to acquire data with as few projections as necessary. The transmitted electrons were energy filtered within $30 \mathrm{eV}$ to remove inelastic scattering signals and the diffraction patterns were recorded with a TVIPS TemCam-XF416. The data was processed using ASTAR (NanoMEGAS SPRL) and Python codes. At each tilt angle a SPED dataset was acquired and grain orientation map calculated. The electron beam was potentially transmitted through multiple grains at each probe position, with each grain constituting information towards the recorded diffraction pattern. As such, multiple grain maps were extracted from each SPED dataset, representing the dominant and auxiliary grains. These complete set of grain orientations from all tilt angles are aligned by calculating the common axis of rotation and then used to further refine the experimental tilt angles, see Figure 1.

Post-processing techniques such as virtual dark-field reconstructions (VDF) selectively highlight specific reflections within the diffraction patterns to reconstruct an image ${ }^{5}$ VDF may be performed from single diffraction reflections or more complicated templates constituted from many reflections. Frozen template VDF, containing many reflections from a simulated diffraction template, were used to highlight individual grains for tomographic reconstruction, such as the two cementite precipitates shown in Figure 2. Whilst VDF may be effectively used to highlight individual features, care must be taken to deconvolve information from overlapping grains which may share common reflections in the diffraction patterns.

In many cases grains contain orientation gradients which exacerbate reconstruction and significantly affect the quality of VDF; however, the latter may be mitigated by actively adapting the VDF template to the local misorientation at each scan pixel. To further address the problem of reconstruction with orientation gradients we utilise grain reconstruction techniques developed in the field of X-ray diffraction imaging ${ }^{6}$, which consider the projected diffraction pattern as a linear combination of local misorientations. Our work highlights development in reconstruction techniques for electron diffraction data towards determining the 3D morphology of individual grains and orientation relationships from a limited number of tilt angles. 

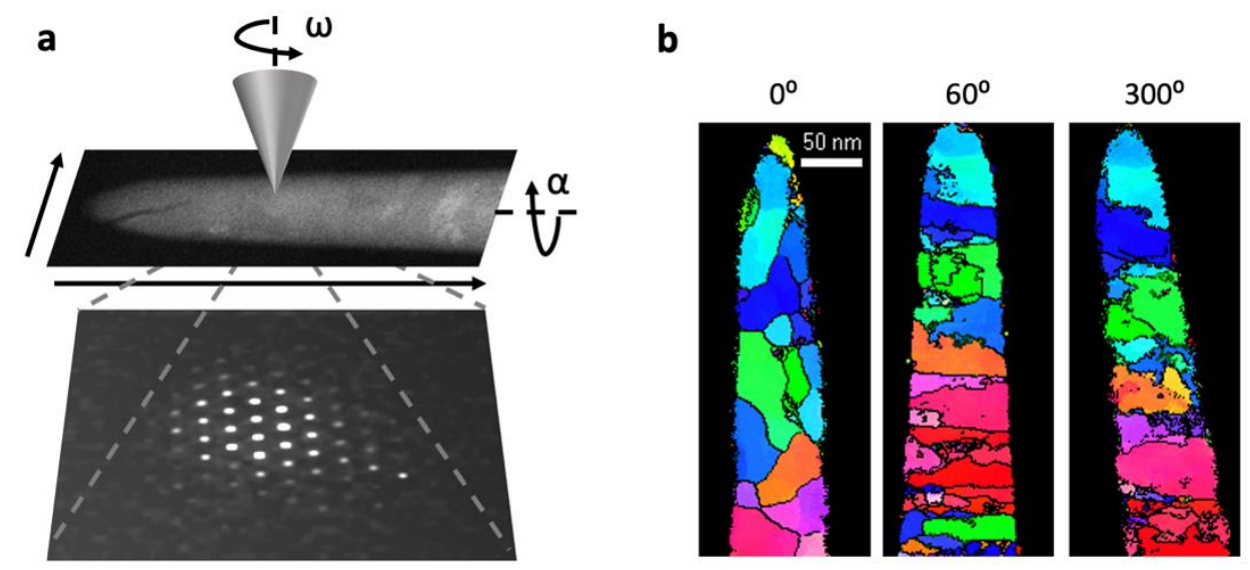

Figure 1. (a) experimental SPED setup: a precessed electron probe is scanned over the sample and a diffraction pattern is acquired at each probe position. (b) the calculated grain orientation maps obtained at different tilt angles are used to further refine the experimental tilt angles but must be aligned and undistorted relative to a reference ADF-STEM image before reconstruction.

$0^{\circ}$

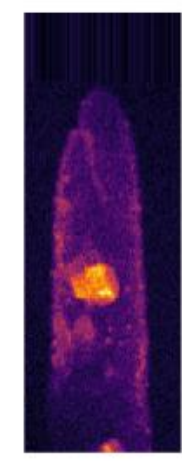

$30^{\circ}$

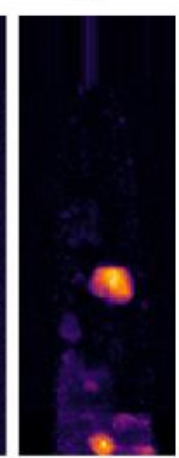

$60^{\circ}$

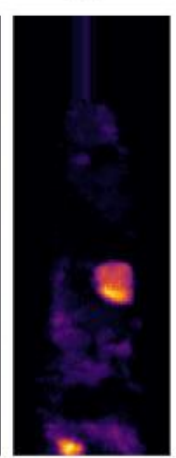

$90^{\circ}$

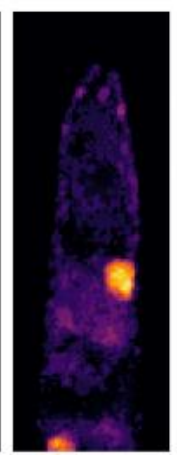

$120^{\circ}$

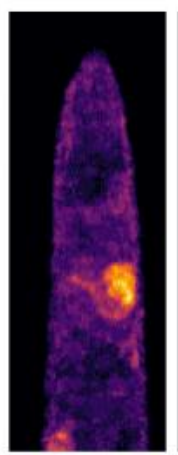

$180^{\circ}$

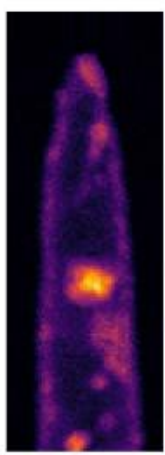

$270^{\circ}$

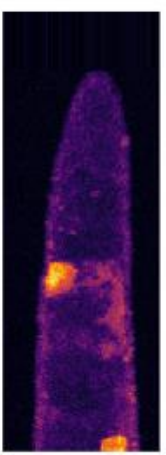

$300^{\circ}$

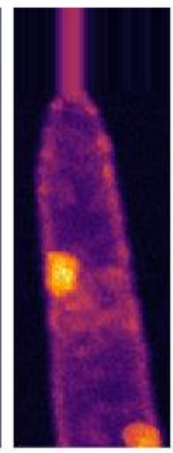

$330^{\circ}$

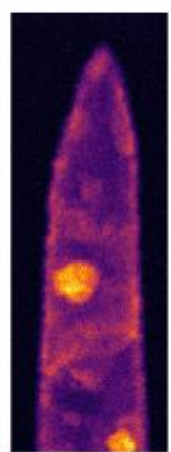

Figure 2. Virtual reconstructions of two cementite grains within an $\mathrm{FeC}$ tip.

\section{References}

1. Rauch, E. F. \& Dupuy, L. Rapid Diffraction Patterns identification through template matching. Archives of Metallurgy and Materials 50, 87-99 (2005).

2. Valery, A., Rauch, E. F., Clément, L. \& Lorut, F. Retrieving overlapping crystals information from TEM nano-beam electron diffraction patterns: ACOM-TEM \& OVERLAPPING CRYSTALS. Journal of Microscopy 268, 208-218 (2017).

3. Eggeman, A. S., Krakow, R. \& Midgley, P. A. Scanning precession electron tomography for threedimensional nanoscale orientation imaging and crystallographic analysis. Nat Commun 6, 7267 (2015).

4. Liu, H. H. et al. Three-Dimensional Orientation Mapping in the Transmission Electron Microscope. Science 332, 833-834 (2011).

5. $\quad$ Meng, Y. \& Zuo, J.-M. Three-dimensional nanostructure determination from a large diffraction data set recorded using scanning electron nanodiffraction. IUCrJ 3, 300-308 (2016).

6. Viganò, N. et al. Three-dimensional full-field X-ray orientation microscopy. Sci Rep 6, 20618 (2016). 